\title{
TOTALLY UMBILICAL CR-SUBMANIFOLDS OF A NEARLY KAEHLER MANIFOLD
}

\author{
S. H. KON AND SIN-LENG TAN
}

\begin{abstract}
The geometry of a CR-submanifold in a Kaehler manifold has been extensively studied. B.Y. Chen has classified the totally umbilical CR-submanifolds of a Kaehler manifold and showed that they are either totally geodesic, or totally real or $\operatorname{dim}\left(D^{\perp}\right)=1$. In this paper we show that such a result is also true in a nearly Kaehler manifold.
\end{abstract}

\section{Introduction}

A. Bejancu [1] introduced the notion of a CR-submanifold of an almost Hermitian manifold. The geometry of a CR-submanifold in a Kaehler manifold has been extensively studied, a number of these results also hold for a CR-submanifold of a nearly Kaehler manifold, see [2], [4], [6] and [7].

B. Y. Chen [4] classified the totally umbilical CR-submanifolds of a Kaehler manifold and showed that they are either totally geodesic, or totally real or $\operatorname{dim}\left(D^{\perp}\right)=1$. In this paper we shall generalize this result to nearly Kaehler manifolds and also show that the anti-holomorphic distribution of a totally umbilical CR-submanifold in a nearly Kaehler manifold is integrable and its leaves are totally geodesic.

\section{Preliminaries}

Let $N$ be an almost Hermitian manifold with almost complex structure $J$ and Hermitian metric $g$. A submanifold $M$ of $N$ is said to be a CR-submanifold of $N$ if there exists a differentiable distribution.

$$
D: x \rightarrow D_{x} \subset T_{x} M
$$

Received October 29, 1994.

1991 Mathematics Subject Classification. 53B35, 53C15, 53C40.

Key words and phrases. CR -submanifolds, nearly Kaehler, totally umbilical. 
on $M$ satisfying the following conditions:

(i) $D$ is holomorphic, i.e., $J\left(D_{x}\right)=D_{x}$ for each $x \in M$.

(ii) the complementary orthogonal distribution

$$
D^{\perp}: x \rightarrow D_{x}^{\perp} \subset T_{x} M
$$

is anti-invariant, i.e., $J\left(D_{x}^{\perp}\right) \subset T_{x} M^{\perp}$ for each $x \in M$.

If $D=\{0\}$, (resp. $D^{\perp}=\{0\}$ ), then $M$ is said to be a totally real (resp. holomorphic) submanifold. The normal bundle $T M^{\perp}$ splits as $T M^{\perp}=J D^{\perp} \oplus \mu$, where $\mu$ is the orthogonal complement of $J D^{\perp}$ and is an invariant subbundle of $T M^{\perp}$ under $J$.

Let $\tilde{\nabla}$ be the Riemannian connection on $N$, then the Gauss and Weingarten formulas are given respectively by

$$
\begin{aligned}
& \tilde{\nabla}_{X} Y=\nabla_{X} Y+h(X, Y), \\
& \tilde{\nabla}_{X} U=-A_{U} X+\nabla_{X}^{\frac{1}{1}} U .
\end{aligned}
$$

for $X, Y \in \Gamma(T M)$ and $U \in \Gamma\left(T M^{\perp}\right)$, where $\nabla$ is the induced Riemannian connection on $M, h$ the second fundamental form of $M, A_{U}$ the second fundamental tensor related by

$$
g\left(A_{U} X, Y\right)=g(h(X, Y), U)
$$

and $\nabla^{\perp}$ is the induced normal connection in the normal bundle $T M^{\perp}$.

A submanifold $M$ is said to be totally umbilical if

$$
h(X, Y)=g(X, Y) H \quad \text { for all } \quad X, Y \in \Gamma(T M),
$$

where $H=\frac{1}{n}$ (trace of $h$ ), called the mean curvature vector of $M$ in $N$. The submanifold $M$ is a totally geodesic submanifold of $N$ if $h(X, Y)=0$ for all $X, Y \in \Gamma(T M)$. It is a minimal submanifold if $H=0$. Obviously a minimal, totally umbilical submanifold is totally geodesic.

A Hermitian manifold $N$ is called a Kaehler manifold if its fundamental 2-form $\Omega$, where $\Omega(X, Y)=g(X, J Y)$ for $X, Y \in \Gamma(T N)$, is closed. It is not difficult to show that an almost Hermitian manifold $N$ is a Kaehler manifold if and only if the almost complex structure $J$ is parallel with respect to $\tilde{\nabla}$, i.e., $\tilde{\nabla}_{X} J=0$ for all $X \in \Gamma(T N)$. An almost Hermitian manifold is called a nearly Kaehler manifold if we have

$$
\left(\tilde{\nabla}_{X} J\right) X=0 \text { for all } X \in \Gamma(T N) \text {. }
$$

Thus, an almost Hermitian manifold $N$ is nearly Kaehler if and only if

$$
\left(\tilde{\nabla}_{X} J\right) Y+\left(\tilde{\nabla}_{Y} J\right) X=0 \text { for all } X, Y \in \Gamma(T N) .
$$




\section{Integrability of the anti-holomorphic distribution of totally umbilical CR-submanifolds}

We will prove in this section an integrability theorem on the anti-holomorphic distribution $D^{\perp}$. We first recall two results on the distribution $D^{\perp}$, see [2, page 28].

Lemma 3.1. (Sato). Let $M$ be a CR-submanifold of a nearly Kaehler manifold $N$. The distribution $D^{\perp}$ is integrable if and only if

$$
g(h(U, X), J W)=g(h(W, X), J U)
$$

for all $U, W \in \Gamma\left(D^{\perp}\right)$ and $X \in \Gamma(D)$.

Lemma 3.2 (Bejancu). Let $M$ be a CR-submanifold of a nearly Kaehler manifold $N$. If $D^{\perp}$ is integrable, then each leaf of $D^{\perp}$ is immersed in $M$ as a totally geodesic submanifold if and only if $g(h(U, X), J W)=0$ for all $U, W \in \Gamma\left(D^{\perp}\right)$ and $X \in \Gamma(D)$.

From the above two lemmas, we are able to obtain the following proposition, whichgeneralizes [3, Lemma 8.2].

Proposition 3.3. Let $M$ be a totally umbilical CR-submanifold of a nearly Kaehler manifold $N$. Then $D^{\perp}$ is integrable and its leaves are totally geodesic in $M$.

Proof. Since $M$ is totally umbilical in $N$, both sides of (3.1) vanish. It follows from the above two lemmas that $D^{\perp}$ is integrable and its leaves are totally geodesic in $M$.

\section{The geometry of totally umbilical CR-submanifolds}

In this section, we will generalize a classification theorem of Chen [4] to nearly Kaehler manifolds. The following generalizes [3; Lemma 7.1].

Proposition 4.1. Let $M$ be a totally umbilical CR-submanifold of a nearly Kaehler manifold $N$. If $\operatorname{dim} D^{\perp}>1$, then we have

(i) $H \perp J D^{\perp}$,

(ii) $A_{J X} Y=0$ for all $X, Y \in \Gamma\left(D^{\perp}\right)$.

Proof. (i) Since $M$ is nearly Kaehler, for $Z, W \in \Gamma\left(D^{\perp}\right)$, we have $\left(\tilde{\nabla}_{Z} J\right) W=$ $-\left(\tilde{\nabla}_{W} J\right) Z$ or $\tilde{\nabla}_{Z} J W-J \tilde{\nabla}_{Z} W=-\tilde{\nabla}_{W} J Z+J \tilde{\nabla}_{W} Z$

or $-A_{J W} Z+\nabla_{Z}^{\frac{1}{}} J W-J \nabla_{Z} W-J h(Z, W)=A_{J Z} W-\nabla_{W}^{\perp} J Z+J \nabla_{W} Z+J h(Z, W)$.

Since $M$ is totally umbilical, we have

$$
J\left(\nabla_{W} Z+\nabla_{Z} W\right)+2 g(Z, W) J H=-A_{J W} Z-A_{J Z} W+\nabla \frac{1}{Z} J W+\nabla_{W}^{\perp} J Z
$$


Hence

$$
\begin{aligned}
& g\left(J\left(\nabla_{W} Z+\nabla_{Z} W\right), Z\right)+2 g(Z, W) g(Z, J H) \\
= & -g\left(A_{J W} Z, Z\right)-g\left(A_{J Z} W, Z\right)+g\left(\nabla^{\perp} J W, Z\right)+g\left(\nabla_{W}^{\perp} J Z, Z\right) .
\end{aligned}
$$

Therefore,

$$
\begin{aligned}
& 2 g(Z, W) g(Z, J H) \\
= & -g(h(Z, Z), J W)-g(h(W, Z), J Z) \\
= & \|Z\|^{2} g(W, J H)+g(Z, W) g(Z, J H),
\end{aligned}
$$

or

$$
g(Z, W) g(Z, J H)=\|Z\|^{2} g(W, J H) .
$$

Interchanging $Z, W$ in the above equation, we obtain

$$
g(Z, W) g(W, J H)=\|W\|^{2} g(Z, J H) .
$$

Hence

$$
g(W, J H)=\frac{g(Z, W)^{2}}{\|Z\|^{2}\|W\|^{2}} g(W, J H) .
$$

Now, if $\operatorname{dim} D^{\perp}>1$, then for $Z$ not parallel with $W$,

$$
g(Z, W)^{2}<\|Z\|^{2}\|W\|^{2}
$$

and so $g(W, J H)=0$, hence $H \perp J D^{\perp}$. (ii) Let $X, Y \in \Gamma\left(D^{\perp}\right)$ and $Z \in \Gamma(T M)$. Then

$$
\begin{aligned}
g\left(A_{J X} Y, Z\right) & =g(h(Y, Z), J X)=g(Y, Z) g(H, J X) \\
& =0 \text { from (i). }
\end{aligned}
$$

Remark. A totally umbilical anti-holomorphic submanifold of a Kaehler manifold is either totally geodesic or a hypersurface [3, Theorem 7.2]. Similarly, if $M$ is a submanifold of a nearly Kaehler manifold $N$ such that $J\left(T_{x} M^{\perp}\right) \subset T_{x} M$ for all $x \in M$, then $M$ can be regarded as a CR-submanifold with $D^{\perp}=J\left(T_{x} M^{\perp}\right)$. For such a manifold, which is also totally umbilical, Proposition 4.1 show that either $M$ is totally geodesic or $M$ is a hypersurface in $N$, generalizing the above mentioned result of Blair and Chen for a totally umbilical anti-holomorphic submanifold of a Kaehler manifold.

The following theorem generalizes Chen's classification theorem [4] to nearly Kaehler manifolds.

Theorem 4.2 Let $M$ be a totally umbilical CR-submanifold of a nearly Kaehler manifold $N$. Then either

(i) $M$ is totally geodesic, or

(ii) $M$ is totally real, or

(iii) $D^{\perp}$ is of dimension 1 . 
Proof. If $\operatorname{dim} D^{\perp}>1$, we have $J H \in \Gamma(\mu)$ from Proposition 4.1. Now if $M$ is not totally real, then $\operatorname{dim} D \geq 2$. Consider $Z \neq 0$ in $\Gamma(D)$, we have

$$
\begin{aligned}
0 & =g(J Z, Z) g(J H, H) \\
& =g\left(A_{J H} J Z, Z\right) \\
& =g\left(-\tilde{\nabla}_{J Z} J H+\nabla_{J Z}^{\perp} J H, Z\right) \\
& =g\left(-\tilde{\nabla}_{J Z} J H, Z\right)=g\left(J H, \tilde{\nabla}_{J Z} Z\right), \quad \text { since } J H \perp Z \\
& =g\left(J H,-\tilde{\nabla}_{J Z} J(J Z)\right) \\
& =g\left(J H,-\left(\tilde{\nabla}_{J Z} J\right) J Z-J \tilde{\nabla}_{J Z} J Z\right) \\
& =g\left(J H,-J \tilde{\nabla}_{J Z} J Z\right), \quad \text { since }\left(\tilde{\nabla}_{J Z} J\right) J Z=0 \\
& =g\left(J H,-J \nabla{ }_{J Z} J Z-J h(J Z, J Z)\right) \\
& =g(J H,-J h(J Z, J Z)) \\
& =-g(J H, J H) g(J Z, J Z)=-\|H\|^{2}\|Z\|^{2}
\end{aligned}
$$

Hence $H=0$. Thus $M$ is minimal and hence totally geodesic in $N$. Finally, for the case $D^{\perp}=\{0\}, M$ is mininal, see [7], and so is totally geodesic.

Remark. A CR-submanifold $M$ with $D^{\perp}$ integrable is said to be $D^{\perp}$-totally umbilical if

$$
h(U, W)=g(U, W) H_{D^{\perp}}
$$

for all $U, W \in \Gamma\left(D^{\perp}\right)$ and some vector field $H_{D^{\perp}}$. A classification theorem, simsilar to Chen's result [4] has been obtained in [5] for $D^{\perp}$-totally umbilical CR-submanifold of a Kaehler manifold. It is easy to show that when $D^{\perp}$ is integrable, such a result is also true in a nearly Kaehler manifold.

\section{References}

[1] A. Bejancu, "CR-submanifolds of a Kaehler manifold I," Proc. Amer. Math. Soc., 69(1978), 135142 .

[2] A. Bejancu, Geometry of CR-submanifolds, Reidel Holland, 1986.

[3] D. E. Blair and B. Y. Chen, "On CR-submanifolds of Hermitian manifolds," Isreal J. Math., 34(1979), 352-363.

[4] B. Y. Chen, "Totally umbilical submanifolds of Kaehler manifolds," Arch. Math., 36(1981), 83-91.

[5] S. M. Khursheed Haider, V. A. Khan and S. I. Husain, "Totally umbilical CR-submanifolds of a Kaehler manifold," Tamkang J. Math., 24(1993), 43-49.

[6] S. H. Kon and Sin-Leng Tan, "CR-submanifolds of a nearly Kaehlerian manifold," Bull. Malaysian Math. Soc., (Second Series), 14(1991), 31-38.

[7] S. H. Kon and Sin-Leng Tan, "CR-submanifolds of a quasi-Kaehler manifold," Tamkang J. Math., 26(1995), 261-266. 